\title{
The Neurophysiological Correlates of Frequency Discrimination and the P300 wave: an ERP study
}

\section{CHRYSI ANASTASAKI ${ }^{1}$}

\author{
${ }^{1}$ UCL Institute of Neurology
}

August 12, 2020

\begin{abstract}
Frequency discrimination is an acclaimed topic of study in cognitive neuroscience since various tones provoke different behavioral and neuropsychological responses. In this study the method of electroencephalography (EEG) was used to extract event-related potentials (ERPs) linked to the P300 component which is associated with the processing of frequency variance. An oddball paradigm was used containing hard and easy deviants embedded with standard tones. The behavioral data indicated shorter reaction times for the easy deviants vs hard deviants. The ERP analysis focused on the P300 waveform across nine electrodes for two types of condition (easyhard). The results indicated that easy targets elicited higher amplitude across parietal electrodes. This report, although completed with a limited sample size, adds up to the discourse about how frequency discrimination is perceived and expressed by behavioral and neurophysiological markers. In line with other studies, the P300 component is found to be involved in the processing of auditory stimuli. The constellation of information invites further research to investigate whether such results are consistent across conditions and different participants.
\end{abstract}

\section{Hosted file}

erp report.pdf available at https://authorea.com/users/320174/articles/475151-theneurophysiological-correlates-of-frequency-discrimination-and-the-p300-wave-an-erp-study 